\title{
NBS1 plays a synergistic role with telomerase in the maintenance of telomeres in Arabidopsis thaliana
}

\author{
Lucie Najdekrova and Jiri Siroky
}

\begin{abstract}
Background: Telomeres, as elaborate nucleo-protein complexes, ensure chromosomal stability. When impaired, the ends of linear chromosomes can be recognised by cellular repair mechanisms as double-strand DNA breaks and can be healed by non-homologous-end-joining activities to produce dicentric chromosomes. During cell divisions, particularly during anaphase, dicentrics can break, thus producing naked chromosome tips susceptible to additional unwanted chromosome fusion. Many telomere-building protein complexes are associated with telomeres to ensure their proper capping function. It has been found however, that a number of repair complexes also contribute to telomere stability.

Results: We used Arabidopsis thaliana to study the possible functions of the DNA repair subunit, NBS1, in telomere homeostasis using knockout nbs 1 mutants. The results showed that although NBS1-deficient plants were viable, lacked any sign of developmental aberration and produced fertile seeds through many generations upon self-fertilisation, plants also missing the functional telomerase (double mutants), rapidly, within three generations, displayed severe developmental defects. Cytogenetic inspection of cycling somatic cells revealed a very early onset of massive genome instability. Molecular methods used for examining the length of telomeres in double homozygous mutants detected much faster telomere shortening than in plants deficient in telomerase gene alone.
\end{abstract}

Conclusions: Our findings suggest that NBS1 acts in concert with telomerase and plays a profound role in plant telomere renewal.

\section{Background}

The ends of linear eukaryotic chromosomes are protected by specific chromatin structures called telomeres that are composed of tandemly repeated telomeric DNA and proteins. In vertebrates, six specific proteins associate with telomeres having affinity to either singlestranded or double-stranded telomeric DNA and they are collectively called shelterin [1]. These complex structures are essential for chromosome stability, as they differentiate chromosome ends from DNA double-strand breaks (DSBs) [2]. They protect chromosome termini from nucleolytic attack and undesirable recombination. Telomeres also counterbalance incomplete replication of terminal DNA by conventional DNA polymerase [3];

\footnotetext{
* Correspondence: siroky@ibp.cz

Department of Plant Developmental Genetics, Institute of Biophysics of the Czech Academy of Sciences, Kralovopolska 135, Brno 61265, Czech Republic
}

cells have evolved specific telomerase reverse transcriptase (TERT), which can synthesise telomeric repeats using its own RNA template thus ensuring proper telomere length. In general, eukaryotic telomeres are composed of tandem G/C rich repeats that end in a single strand 3' overhang which can fold back and invade the duplex repeats to form the so-called T-loop [4]. In the absence of telomerase, telomeres become non-functional, shorten with successive cell divisions, and chromosome termini can fuse as a consequence of de-protection. Their fusion is a result of the non-homologous-end-joining (NHEJ) which is the prevailing mechanism of DSB healing in plants. Although numerous attempts have been made to assess shelterin counterparts in plants, to date these proteins have not been identified (see [5] for review). Together with proteins invariantly occurring at the telomeres and providing a telomere "capping" function, many additional protein complexes are regularly found in eukaryotic 
telomeres. Paradoxically, many of these proteins are involved in DNA repair or recombination and this seems inappropriate at the telomere ends which should be hidden from recombination events and end-to-end fusions.

Over the past decade, an increasing body of data has accumulated on the concerted network of DNA repair factors and various protein kinases following the disruption of DNA integrity, including noxious extrinsic factors, DNA replication errors, checkpoint signalling and, meiotic and somatic recombination. One system that plays an essential role in DNA repair, recombination, DNA replication as well as in the cell cycle checkpoint activation and telomere maintenance, is the MRN complex (for recent reviews see [6,7]). This consists of three subunits (MRE11RAD50-NBS1). A single NBS1 molecule is associated with two dimers of MRE11 and RAD50. The MRE11 and RAD50 proteins form a heterotetramer which contains two DNA-binding and processing domains that can bridge free DNA ends [8]. In Saccharomyces cerevisiae, this complex comprises subunits MRE11, RAD50, and XRS2. Whereas both proteins, MRE11 and RAD50, share a high homology across various eukaryotes, the XRS2 protein has a lower degree of homology with NBS1, which is specific for mammals and plants [9], although a functional homologue of NBS1 has been found in Schizosaccharomyses pombe [10].

In humans, mutation in the NBS1 gene leads to the chromosomal instability disorder, Nijmegen breakage syndrome 1 . Besides other clinical hallmarks, this syndrome is associated with enhanced sensitivity to ionizing radiation and chromosomal instability which leads to early developing cancer even in $\mathrm{NBS1}^{+/-}$heterozygotes [11]. Murine $N B S 1^{+/-}$heterozygotes are phenotypically normal although complete removal of the NBS1 is embryonically lethal in mice [12]. Accumulating evidence demonstrates that NBS1 interacts with telomeres and contributes to their stability, at least in human and mouse cells (reviewed in [11]). Direct interaction of NBS1with telomere repeat-binding factor 1, TRF1, has been shown for immortalized telomerase negative cells [13] implying that this interaction might be involved in the alternative lengthening of telomeres. Moreover, it has been shown by indirect immuno-fluorescence that NBS1 co-localise with a shelterin constituent, telomere repeat-binding factor 2 (TRF2), during the $S$ phase in cultured HeLa cells [14], possibly by modulating t-loop formation. Similarly, in mouse embryonal fibroblasts, active recruitment of NBS1 to dysfunctional telomeres has been observed [15].

It is known that MRE11 and RAD50 together with protein kinases ATM and ATR, are also essential for proper telomere maintenance in plants (Reviewed in $[5,16,17]$ ). Inactivation of Arabidopsis RAD50 or MRE11 leads to hypersensitivity to ionizing radiation or radiomimetics and reduced plant health and even sterility. In knockout RAD50 or MRE11 mutant Arabidopsis plants genome instabilities are induced, including chromosome end-to-end fusions [18-21]. Absence of RAD50 led to rapid shortening of telomeres and loss of telomere repeats accompanied by chromosome-end fusions, while in double mutant plants (rad50/tert) a synergistic effects of RAD50 and telomerase on the frequency of bridges have been found [19], demonstrating the dual role of the RAD50 protein in plants. A homolog of the third MRN constituent, NBS1, has been isolated in the higher plants, Arabidopsis thaliana and Oryza sativa [22]. The NBS1 proteins from both plant species were shown to be smaller in size than animal NBS1, but both contained typical domains such as the FHA (forkhead-associated), BRCT (BRCA1 C Terminus) domain, the MRE11-binding domain, and the ATMinteracting domain. Functional analysis using yeast twohybrid assay showed that the OsNBS1 protein interacted not only with plant MRE11 but also with animal MRE11. OsNBS1 mRNA expression was found to be higher in the shoot apex and young flower and AtNBS1 expression increased when plants were exposed to X-rays [22]. Cytogenetic analyses showed numerous anomalies including the fragmentation of meiotic chromosomes in At $n b s 1$ knockout mutants [23], although on simultaneous inactivation of plant ATM.

In this study, we examined the role of NBS1 in telomere maintenance in the plant model species Arabidopsis thaliana. Using plants deficient in both genes, NBS1 and TERT, we found that plants exhibited severe genomic instability even in early generations. These phenotypes worsened with increasing generations on selfpollination and plants developed serious developmental defects leading to sterility in the $6^{\text {th }}$ generation. By comparing the length of telomeres in double and single mutants we observed accelerated and more frequent telomere shortening in the former.

\section{Results}

tert/nbs 1 mutants displayed severe morphologic developmental defects over increasing generations

We performed a crossing between nbs1 and tert homozygous plants. From segregating F2 seedlings, wild-type plants, nbs1 mutants, two independent tert lines 69-1 and 69-2, as well as double homozygotes were selected by genotyping using T-DNA-specific and gene-specific primers. Two independent tert/nbs1 lines were selected for onward analyses, K12 and L2. These lines are further referred to as generation 1 (G1). Upon self pollination, we obtained five consecutive generations, G2 to G6, until plants displayed defective morphology (Figure 1B), produced infertile seeds (Figure 2A), and were incapable of subsequent cultivation. Siliques from G5 plants, both lines, 


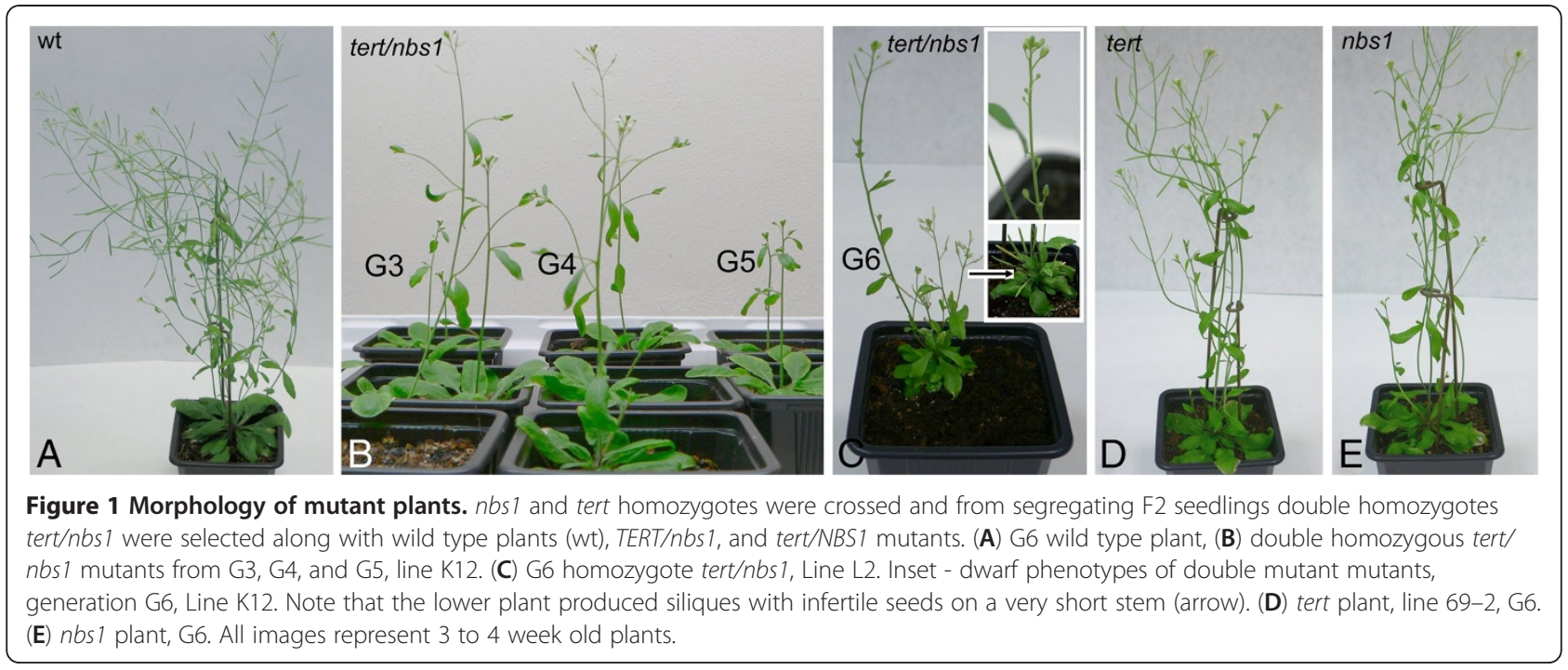

K12 and L2, contained only $12 \pm 4$ seeds with a $45 \%$ fertility. Although the number of seed pods from G6 plants was the same as in G5 lines, seeds failed to germinate in either soil or MS agar cultures (Figure 2D). By contrast, single tert mutant seeds exhibited no signs of fitness derogation from G2 to G6 in either line 69-1 or 69-2 (Figure 2B).

\section{Genomic instability of tert/nbs 1 is accelerated in comparison to single tert mutants}

We predicted accelerated signs of genome instability in somatic tissues of double homozygotes in comparison with single tert mutants. Arabidopsis tert mutants are known to be gradually more and more prone to chromosome fusions due to telomere de-protection in successive generations [24] with massive onset of fused chromosomes from the $6^{\text {th }}$ generation on. In our experiments, fused chromosomes were easily detected as anaphase bridges (Figure 3B-D). We used statistics [25] to compare the frequencies of anaphase bridges. Consistent with findings [24,26,27] single tert mutants appeared to possess $5.4 \%$ of anaphase bridges (Figure 3F) in G6 and increased incidence of bridges in $\mathrm{G} 7$ to $7.6 \%$ (Figure $3 \mathrm{G}$ ).

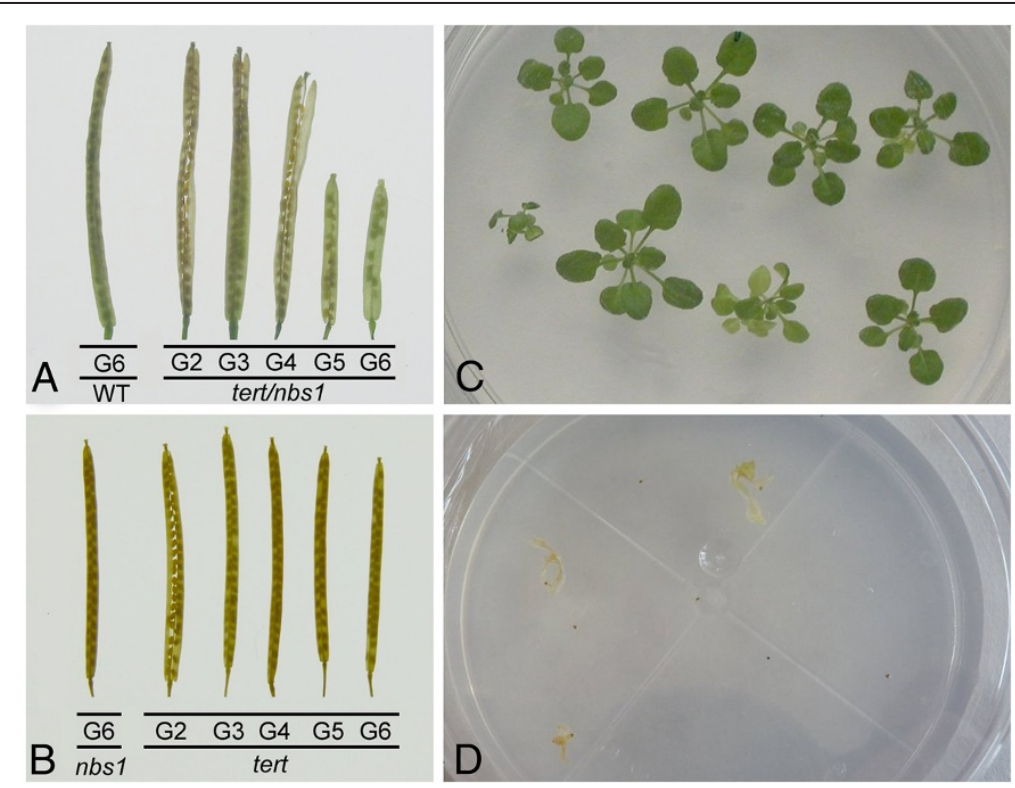

Figure 2 Seed production and germination of late generation double mutants. (A) Comparison of siliques from wt (G6) and G2 to G6 double homozygotes tert/nbs1, Line K12. Seeds from G5 plants were $45 \%$ sterile in both lines, K12 and L2. G6 seeds were completely sterile. (B) nbs1 siliques (G6) compared to tert, line 69-2 (G2 to G6). The fertility of nbs1 and tert seeds was almost $100 \%$, irrespective of seed generation. (C-D) Seedlings of G7 plants on agar MS cultures, line K12. (C) Heterozygote TERT/nbs1. (D) tert/nbs1 genotype seedlings did not germinate. 

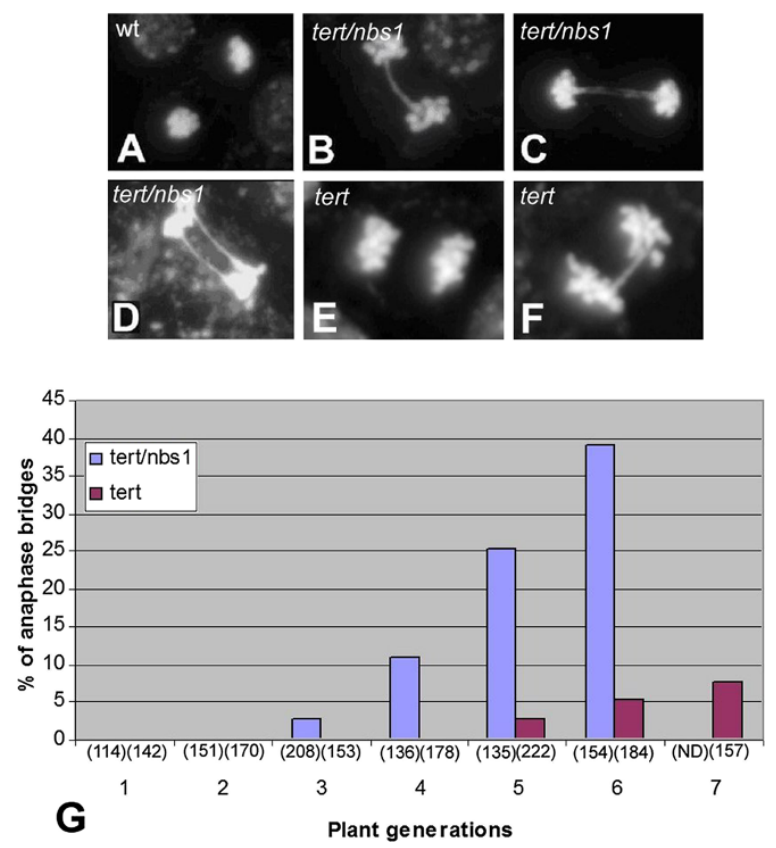

Figure 3 Genomic instability of tert/nbs 1 manifested as mitotic anaphase bridges. Pistils of experimental plants were treated for microscopy, tissues fixed, squashed and stained with 4',6-diamidino2-phenylindole (DAPI). (A) Anaphase from G6 wild type plant, (B-D) anaphases from double homozygous tert/nbs1, line K12, generation G6 possessing single anaphase bridge, twin bridge and two single bridges, respectively. (E-F) Anaphases from single homozygous tert plants, line 69-2, generation G6. Whereas in this tert seed generation, the majority of anaphases exhibited no bridge (E), bridged anaphases were detected in $5.4 \%$ (F). (G) The frequency of bridges containing anaphases regardless of number of bridges between the respective anaphases are shown for tert/nbs 1, line L2, (blue columns) and tert, line 69-2, (purple columns) in consecutive generations. The numbers of total anaphases scored are indicated in parentheses. No bridged anaphases were detected in nbs 1

heterozygotes or wild-type plants. The 95\% Confidence Intervals [25] show significant differences between the values for generations $\mathrm{G} 3$ to $\mathrm{G} 6$. (ND = due to severe developmental defects, no plants were available for cytology).

In comparison, double mutants tert/nbs1 started to develop mitotic anaphase bridges from the G3, reaching 39.4 and $38.1 \%$ in lines $\mathrm{K} 12$ and $\mathrm{L} 2$ respectively, in generations G6 (Table 1). Microscopic inspection disclosed no anaphase bridges in $n b s 1$ generations, as in wild-type plants (TERT/NBS1) selected during the genotyping process.

\section{Meiotic defects in tert/nbs 1 mutants}

Although microscopic inspection revealed no aberrations in male meiocytes from wild-type plants and $n b s 1$ mutants, double mutants (tert/nbs1) displayed numerous chromosome bridges between bivalents in anaphase I and between univalents in anaphase II (Figure 4A-D). These bridges were observable in preparations from pollen mother cells from G2 on. No other signs of meiotic aberrations were observed. Pachytene bivalents were fully aligned and male meiosis ended by morphologic perfect tetrads. Pollen viability of tert/nbs1 was 100\% (Figure 4I). From the analysis of tert mutants from G6, among 55 individual anaphase I figures scored, both lines 69-1 and 69-2, only two contained bridged bivalents (Figure 4F). Products of male meiosis were apparently normal for tert G6 plants (Figure 4G). Comparing aceto-carmine stained pollen grains in anthers, no differences between genotypes tested were noted and all pollen grains were apparently fertile (Figure $4 \mathrm{H}-\mathrm{J}$ ).

\section{Chromosomal fusion points of mutant cells exclusively involve chromosome termini}

In order to judge whether terminal or intercalary sites of chromosomes are involved in fusion sites of tert/nbs 1 mutants, we performed repeated rounds of bicolour FISH with chromosome-specific BAC probes directly adjacent to Arabidopsis telomeres as in [26]. On anaphase bridges of tert/nbs1 mutants, we almost exclusively found signals from chromosome termini (Figure 5B-D). Using FISH with a directly labelled PNA telomere probe, we detected telomere tracts on a portion of bridges in G3 in both lines (Table 2), K12 (Figure 5F) and L2. From generation G4 on, telomere signals were not detected on the anaphase bridges of the double mutants (Figure $5 \mathrm{H}$ ). This finding can be explained by the shortening of the telomere to an extent not detectable by the in situ hybridization procedure, consistent with our assumption that telomeres of the tert/nbs 1 mutants shorten considerably faster than these of plants with the knockout telomerase gene only. The anaphase bridges of the tert mutants (at least for generation G6) also contained FISH signals from telomere-adjacent BAC probes or chromosome terminal sequences as rDNAs (Figure 5J-L). As a rule, fused tert chromosomes contained telomerespecific signals in generations G5 and G6 (Table 2, Figure $5 \mathrm{~N}$ and $\mathrm{P}$ ).

From examination of the possible participation of respective chromosome ends forming anaphase bridges in tert/nbs 1 double mutants, we found a notable bias to fusions of the chromosome 2 right arm (Figure 6, Table 3). This more frequent involvement of $2 \mathrm{R}$ chromosome ends in fusions, pertaining exclusively to mutant line K12, was observable from G3 throughout G6. In tert mutants, both lines, 69-1 and 69-2, and tert/nbs 1 of the L2 line anaphase bridges basically carried random terminal signals.

\section{Telomeres of tert/nbs 1 plants rapidly shorten and to a greater extent than the tert lines}

To assess the overall lengths of telomere tracts in somatic cells of the tert/nbs1 mutants, we applied the TRF 
Table 1 The frequency of anaphase bridges in tert/nbs 1 Arabidopsis mutants during cultivation

\begin{tabular}{|c|c|c|c|c|c|c|c|}
\hline \multirow[t]{2}{*}{ Generation } & \multirow[t]{2}{*}{ Line } & \multirow{2}{*}{$\begin{array}{l}\text { Anaphases } \\
\text { scored }\end{array}$} & \multicolumn{4}{|c|}{ No of anaphase bridges } & \multirow{2}{*}{$\begin{array}{c}\% \text { total } \\
\text { bridged } \\
\text { anaphases }\end{array}$} \\
\hline & & & Single & Double & Triple & Quadruple & \\
\hline G1 & $\mathrm{K} 12$ & 100 & & & & & 0.0 \\
\hline G1 & L2 & 131 & & & & & 0.0 \\
\hline G2 & $\mathrm{K} 12$ & 112 & & & & & 0.0 \\
\hline $\mathrm{G} 2$ & L2 & 91 & & & & & 0.0 \\
\hline G3 & $\mathrm{K} 12$ & 95 & 4 & & & & 4.2 \\
\hline G3 & L2 & 357 & 6 & 3 & & & 2.5 \\
\hline G4 & $\mathrm{K} 12$ & 248 & 25 & 4 & & & 11.7 \\
\hline G4 & L2 & 42 & 3 & & & & 7.1 \\
\hline G5 & $\mathrm{K} 12$ & 348 & 57 & 28 & 1 & & 22.1 \\
\hline G5 & $\mathrm{L} 2$ & 331 & 53 & 32 & & & 25.7 \\
\hline G6 & $\mathrm{K} 12$ & 614 & 130 & 101 & 10 & 2 & 39.4 \\
\hline G6 & L2 & 381 & 67 & 59 & 16 & 3 & 38.1 \\
\hline
\end{tabular}

In G1 to G2 no anaphase bridges were detected. Two independent lines of tert/nbs1 double mutants were scored, K12 and L2. The 95 \% Confidence Intervals [25] show that no statistical differences exist between the values for lines K12 and L2 in generations G3 to G6.

method (terminal restriction fragment analysis) to DNAs isolated from individual plants of various generations and from both lines, K12 and L2. Whereas wild-type Arabidopsis thaliana of the Columbia ecotype possess telomeres with lengths ranging from 2 to $5 \mathrm{~kb}$, mutant lines displayed remarkable shortening. When comparing tert and tert/nbs1, the telomeres of the latter shortened markedly faster (Figure 7). In contrast to the wild-type, we found in both mutants banded patterns where individual bands represent individual telomere lengths ranging from 4 to $1 \mathrm{~kb}$ for both tert and tert/nbs1 in G1. With successive generations of plants, the length differences were more apparent, reaching 1.5 to $0.7 \mathrm{~kb}$ for tert of G6 plants, line 69-2, and as little as $0.6 \mathrm{~kb}$ for tert/
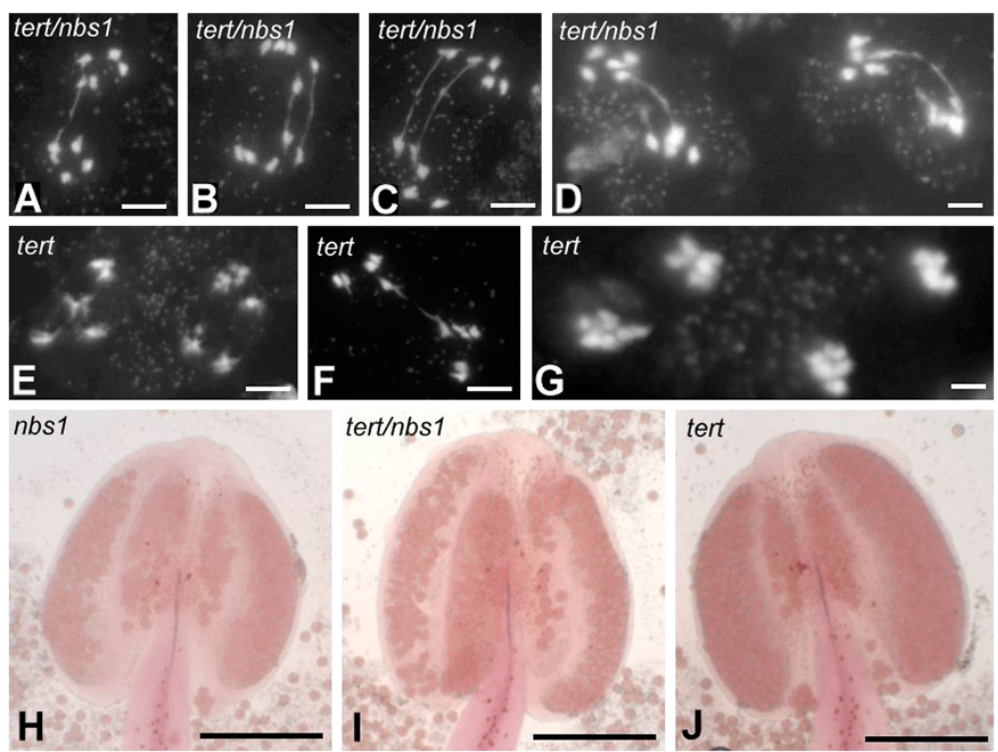

tert/nbs1
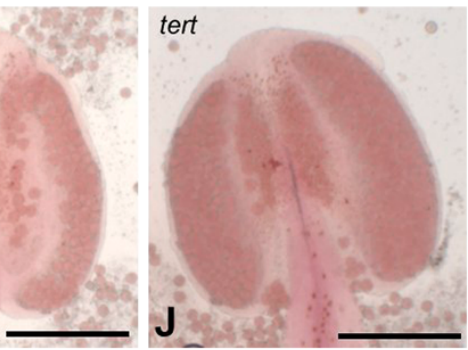

Figure 4 Defects in male meiosis. (A-C) Meiotic anaphases I in double homozygous tert/nbs 1 pollen mother cells, line K12, G2 generation. Bivalents are connected with one (A) or two bridges (B, C). (D) During anaphase II, segregating univalents are interconnected by bridges as a consequence of chromatid fusion in earlier phases of meiosis. Note the coequal pattern of dividing univalents. (E-G) tert mutants, line 69-2, G6. (E) In the majority of anaphase I preparations, no bridges were presented. (F) A bridge detected in anaphase 1 of tert, line 69-2, G6. (G) tert products of anaphase II are morphologically normal univalents (line 69-2, G6). (H-J) Anthers from G6 plants stained with aceto-carmine. (H) nbs 1, (I) tert/nbs 1, and (J) tert. Bars indicate $10 \mu \mathrm{m}$ for $(\mathbf{A}-\mathbf{G})$ and $200 \mu \mathrm{m}$ for $(\mathbf{H}-\mathbf{J})$. 

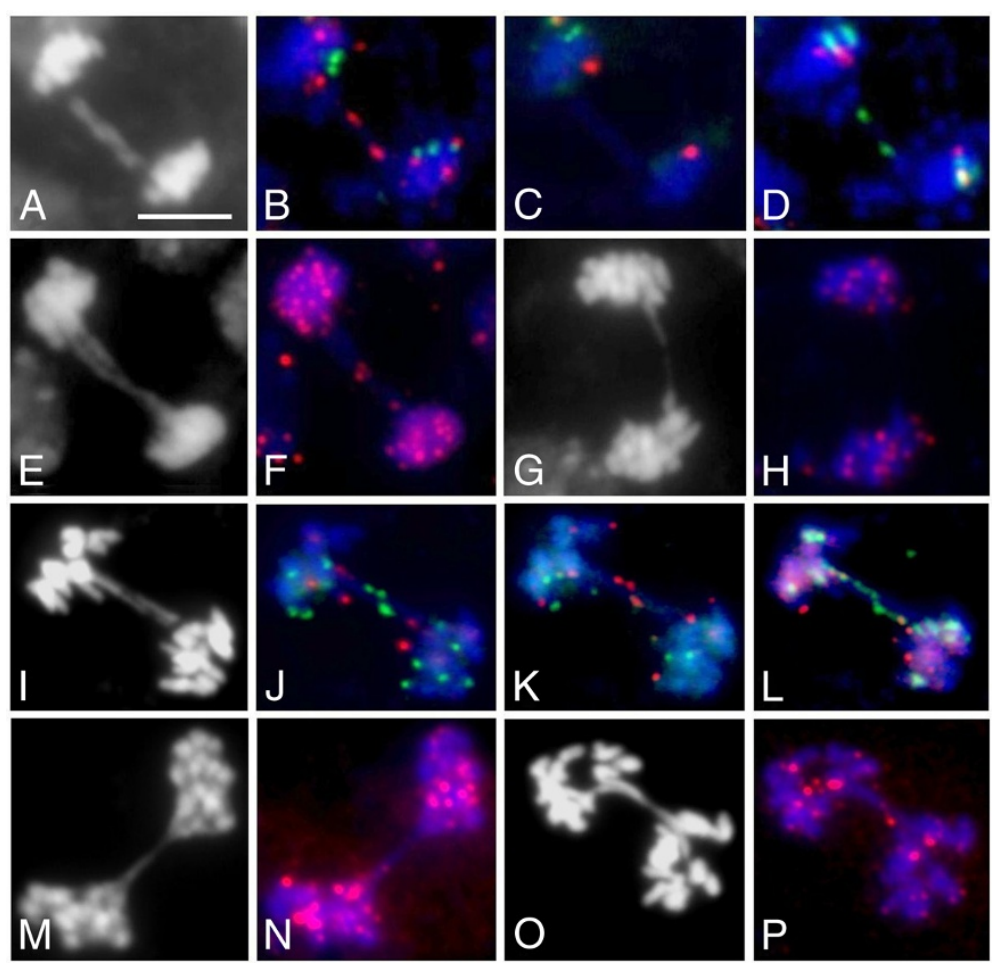

Figure $\mathbf{5}$ Chromosome fusion points as revealed by FISH. (A-H) tert/nbs 1 double homozygotes, (I-P) tert. (A-D) tert/nbs 1 mutant, generation G6, line K12. Sequential FISH on mitotic anaphase bridge revealed involvement of the right ends from chromosome 1. (A) DAPI stained anaphase with the bridge consisting of two chromatids. (B) The signals from BAC probe F5I6 (Cy3 labelled, red) are presented on both bridges. In the second FISH (C) no signals on the bridges are presented whereas in (D) the green probe (SpectrumGreen labelled, BAC F5I6) highlights the same positions as in (B). According to the FISH setup, alternation of colours unambiguously disclosed the fusion of the $1 \mathrm{R}$ chromosome end. (E-H) FISH with telomere-specific probe. Using telomere-specific Cy3-labelled PNA-C $\mathrm{TA}_{3}$-probe, fusion points were detected on two chromatid anaphase bridges from line K12, generation G3 plants (F), whereas in higher generations (G4) the signals from PNA-probe were not detectable on the anaphase bridges $(\mathbf{H})$. To demonstrate the morphology of bridges DAPI stained images are presented (E, G). (I) DAPI stained anaphase displaying twin anaphase bridges of tert mutant, line 69-2, generation G6. (J-L) Sequential FISH. (J, K) Left ends of chromosomes 3 were sequentially labelled by 3 L-specific T4P13 BAC, green in first FISH (J), and red in second FISH (K). In the third FISH, green signals from the probe for 45S rDNA illuminated the $2 \mathrm{~L}$ chromosome end (L). Thus, direct reciprocal fusions of $3 \mathrm{~L}$ and $2 \mathrm{~L}$ chromosome ends were proven. (M-P) Anaphase bridges from tert mutants probed by telomere PNA probe. (N) tert line 69-1, G5 and (P) line 69-2, generation G6. In both lines fusion points were decorated by telomere-specific probes from the generation $\mathrm{G} 5 \mathrm{on}$. (M, $\mathbf{0})$ DAPI stained anaphases with bridges. Bar represents $10 \mu \mathrm{m}$ for all images.

nbs1 G6 plants (Figure 7A, arrowhead). The trend of the accelerated telomere shortening of tert/nbs 1 double mutants is also visible when comparing G4 to G5 plants (Figure 7B). Homozygous nbs1 plants displayed no marks of telomere shortening in the same experiments.

\section{Discussion}

\section{Experimental setup and plant morphology}

Individual constituents of the MRN plant complex have been stepwise analyzed in recent years. MRE11 T-DNA insertion mutants have been described as having severe impact on plant growth and the fertility of homozygotes [20,28]. Similarly, mutant Arabidopsis plants with TDNA within a RAD50 gene were sterile $[18,29,30]$ and capable of survival only as in vitro cultures.
In this study, we analyzed the possible role of NBS1 in telomere length homeostasis. We used the same Arabidopsis mutant line GK-570B09 as in [23]. T-DNA insertion resulted in $\mathrm{N}$-terminal truncated protein lacking functional FHA and BRCT domains, whereas the MRE11 binding domain was retained. This deficiency did not affect the growth or fertility of the $n b s 1 / n b s 1$ homozygotes (Figure 1), as in [23]. Homozygous plants deficient in TERT gene have been extensively studied $[24,26,31,32]$ and shown to possess no telomerase activity [31]. Nevertheless, the plants were viable in our experiments and provided fertile seeds over seven generations, similar to [31]. By contrast, double homozygous tert/nbs 1 plants exhibited signs of developmental impairment from the G3 generation (Figure 1B), accompanied by gradual decline in seed production (Figure 2A). 
Table 2 Comparison of telomeric signals on anaphase bridges of tert/nbs 1 and tert mitoses using FISH with telomerespecific PNA probe

\begin{tabular}{|c|c|c|c|c|c|c|c|}
\hline \multicolumn{4}{|c|}{ tert/nbs1 } & \multicolumn{4}{|c|}{ tert } \\
\hline Generation & Line & Bridges & Telomeric signals & Generation & Line & Bridges & Telomeric signals \\
\hline G3 & K12 & 4 & 4 & G3 & $69-1$ & 0 & ND \\
\hline G3 & L2 & 9 & 7 & G3 & $69-2$ & 0 & ND \\
\hline G4 & K12 & 29 & 0 & G4 & $69-1$ & 0 & ND \\
\hline G4 & L2 & 0 & ND & G4 & $69-2$ & 0 & ND \\
\hline G5 & K12 & 45 & 0 & G5 & $69-1$ & 4 & 4 \\
\hline G5 & L2 & 65 & 0 & G5 & $69-2$ & 3 & 3 \\
\hline G6 & K12 & 80 & 0 & G6 & $69-1$ & 10 & 10 \\
\hline G6 & L2 & 47 & 0 & G6 & $69-2$ & 12 & 10 \\
\hline
\end{tabular}

K12 and L2 represent independent lines of tert/nbs1 mutants, whereas 69-1 and 69-2 are two independent tert mutants. ND applies to when no bridged anaphases were available and no FISH was performed. In tert/nbs 1 anaphase bridges were detected from G3 on, in both lines; in the majority of fused anaphase bridges telomere signals were detected. Nevertheless, from generation G4, telomeric signals were no longer detected. Anaphase bridges in tert mutants started to occur from $\mathrm{G} 5$ in both lines, and in a prevailing manner the bridges bore telomere-specific signals.

Although the dwarf G6 plants provided seeds, they had no germination capacity (Figure 2D).

\section{Genomic instability of the tert/nbs 1 homozygotes}

The measure of genomic instability in our experiments was the occurrence of mitotic anaphase bridges (Figure 3B-D). Whereas in wild-type plants, anaphase bridges were not detected, yet in double homozygotes tert/nbs 1 the presence of anaphase bridges was detected from G3 on (Figure 3G). Over the next three seed generations (G6 was terminal), the percentage of bridged anaphases increased dramatically, reaching almost $40 \%$ in G6 (Figure 3).

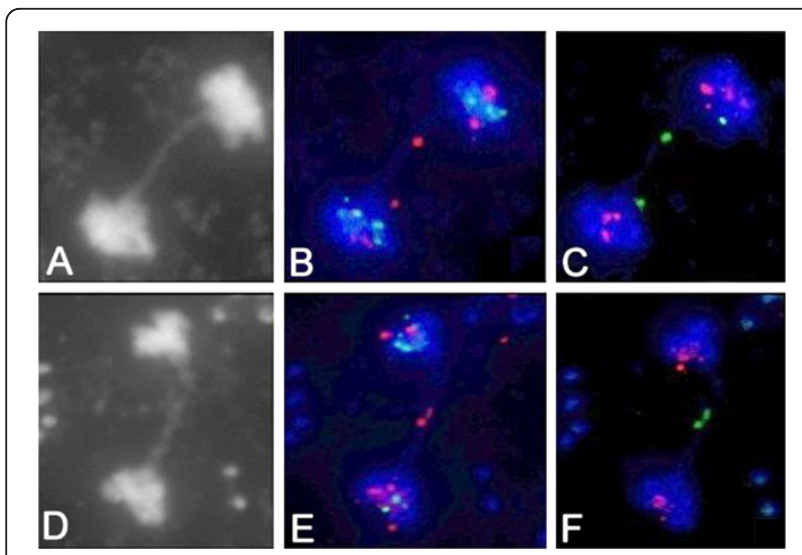

Figure 6 Biased chromosome fusions. (A-F) Anaphase bridges represent the involvement of the right arm of chromosome 2. Sequential FISH with telomere-adjacent 2R specific BAC clone revealed the fusion of $2 R$ chromosome ends. The morphology of bridges is apparent from DAPI stained images (A, D). Using consecutively BAC F11L15 labelled with Cy3 (red, B and E) in the first FISH and labelled with SpectrumGreen (green, $\mathbf{C}$ and $\mathbf{F}$ ), the direct contact of chromosome termini is highlighted. This respective alternation of signals during sequential FISH procedures was detected in the great majority of anaphase bridges tested in line K12. (A-C) Generation G3, (D-F) Generation G6.
Comparing the two independent tert/nbs1 lines, K12 and L2, no difference in frequency of bridges was found (Table 1). In contrast, tert mutants began to exhibit anaphase bridges in $\mathrm{G5}$, but the proportion remained relatively low: $2.7 \%, 5.4 \%$, and $7.6 \%$ for G5, G6, and G7 (Figure 3G). Since nbs1 homozygotes did not display any anaphase bridge under microscopic evaluation, markedly enhanced frequency and accelerated occurrence of anaphase bridges observed in our double homozygotes can only be explained by the synergistic interaction of both deficiencies.

We also detected anaphase bridges in meiotic anaphases of pollen mother cells of the double mutants (Figure 4) while other stages of meiosis were apparently normal. We observed no defective meiosis of single $n b s 1$ mutants across any generations studied. Employing this identical $n b s 1$ mutant but in an atm background, massive defects in early stages of meiosis occurred, implying a role for NBS1 in the control of meiotic progression which was independent of ATM signaling [23].

\section{Fused chromosomes of tert/nbs 1 entirely involved} chromosomal termini

The fusion of chromosomes by the NHEJ mechanism is a consequence of telomere uncapping and can be the result of random fusions of various replicated chromosomes or the consequence of the fusion of sister chromatids of the same chromosome [27]. FISH analysis with BAC probes originating from chromosomal sites directly adjacent to telomeres of Arabidopsis chromosomes revealed that the majority of bridges contained terminal BAC signals (Figure 5B-D) and, specifically, no combination of signals from various chromosomes. Therefore the prevalent mechanism of chromosomal joining was the fusion of sister chromatids. This is in contrast to tert mutants, where various chromosome termini were randomly recruited to 
Table 3 Fusions of the right arms of chromosome 2 of tert/nbs 1 and tert mutant Arabidopsis plants as revealed by FISH

\begin{tabular}{|c|c|c|c|c|c|c|c|c|c|}
\hline \multicolumn{5}{|c|}{ tert/nbs1 } & \multicolumn{5}{|c|}{ tert } \\
\hline Gen. & Line & $\begin{array}{c}\text { Anaphase } \\
\text { bridges tested } \\
\text { by FISH }\end{array}$ & 2R signals & 2R signals (\%) & Gen. & Line & $\begin{array}{c}\text { Anaphase } \\
\text { bridges tested } \\
\text { by FISH }\end{array}$ & $2 \mathrm{R}$ signals & 2R signals (\%) \\
\hline G3 & $\mathrm{K} 12$ & 36 & 26 & 72.2 & G3 & 69-1 & 0 & ND & ND \\
\hline G3 & L2 & 41 & 0 & 0.0 & G3 & $69-2$ & 0 & ND & ND \\
\hline G4 & $\mathrm{K} 12$ & 40 & 18 & 45.0 & G4 & $69-1$ & 0 & ND & ND \\
\hline G4 & L2 & 45 & 3 & 6.7 & G4 & $69-2$ & 0 & ND & ND \\
\hline G5 & $\mathrm{K} 12$ & 32 & 27 & 84.4 & G5 & $69-1$ & 6 & 0 & 0.0 \\
\hline G5 & L2 & 37 & 0 & 0.0 & G5 & $69-2$ & 5 & 0 & 0.0 \\
\hline G6 & $\mathrm{K} 12$ & 42 & 33 & 78.6 & G6 & $69-1$ & 10 & 1 & 10.0 \\
\hline G6 & L2 & 54 & 2 & 3.7 & G6 & $69-2$ & 12 & 0 & 0.0 \\
\hline
\end{tabular}

Three sequential FISH with chromosome-end specific probes revealed enhanced occurrence of $2 \mathrm{R}$ chromosome ends in line $\mathrm{K} 12$ of the tert/nbs 1 double mutants. In the sibling line $\mathrm{K} 2$, the fusion points were represented by random chromosome-arm interactions. In tert mutants lines 69-1 and 69-2, the occurrence of anaphase bridges starts from G5 on. Although the number of anaphase bridges available for analyses was rather low, cf. Figure 3G, only in one case the 2R-2R fusion had been detected. ND - no anaphases available.

fusions in both lines, 69-1 and 69-2 (Figure 5J-L). In the sparsely occurring bridges of G3 of double homozygotes, we were able to observe clear telomere signals on the bridges in a high proportion (Figure 5F, Table 2) which was in striking contrast to higher generations (G4 to G6) where telomere tracts on the anaphase bridges were never detected (Figure $5 \mathrm{H}$ ). In contrast, tert mutants regularly contained traces of telomere sequences, at least in G5 and G6 (Figure 5N and P; Table 2). The loss of telomere DNA has been noticed on the mitotic bridges of the Arabidopsis rad50 mutant [19]. However, double hetrozygotes tert/ rad50 frequently contained the telomere repeats [19], thus

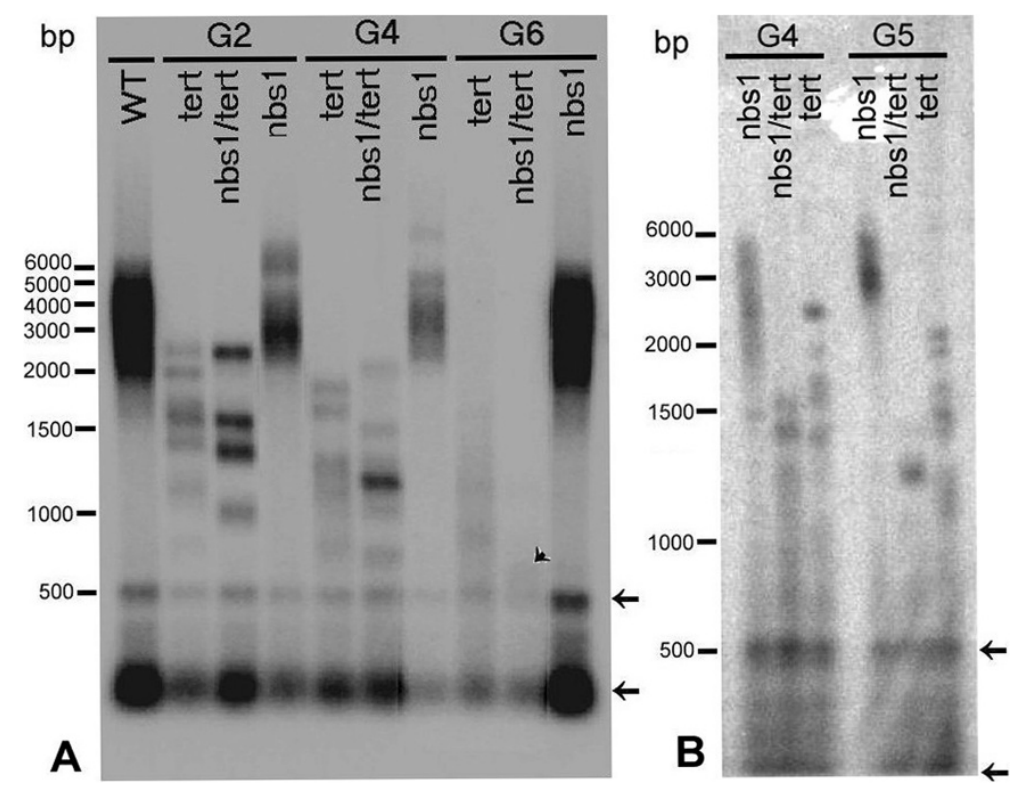

Figure 7 TRF analysis of mutant plants. Terminal telomere fragments were obtained by digestion of DNA isolated from representative plants in second, fourth, and terminal generations (G2, G4, G6) by Tru1l restriction endonuclease, separated on 1\% agarose gel, Southern transferred, and visualized by ${ }^{32} \mathrm{P}$ labelled $\mathrm{C}_{3} \mathrm{TA}_{3}$ probe. (A) Wild type and nbs 1 mutants had telomere lengths 5000 to $2000 \mathrm{bp}$. Gradual shortening of tert mutants (line 69-2) is accompanied by more dramatic telomere shortening in double homozygotes tert/nbs 1, (line K12) to as short as 600 bp in G6 (arrowhead). The differences in the intensity of signals reflect slight variations in the amount of DNA loaded in each lane. (B) The comparison of TRF fragments of plants G4 and G5. Gradual decline in the length of the fragments of double homozygotes tert/nbs1 (line L2) from 1600-700 bp to 1300-700 bp is evident between consecutive generations. The ranges of the lengths are markedly shorter than for tert (line 69-1) displaying 2500-1400 bp and 2000-1000 bp respectively for generations G4 and G5. Arrows point to the interstitial telomere sequences in (A) and (B). 
arguing for two different roles of the RAD50 in either the de-protection of telomeres or with telomeres already shortened by the absence of TERT.

In a number of Arabidopsis mutants exhibiting somatic anaphase bridges the participation of either the homologous chromosomes or one-chromatid fusions has frequently been described; this holds for mre11-3/ku70 mutant (44\% of FISH-identified fusions were decorated by a single telomere-adjacent probe) [20], or for atm/tert mutants where a single chromosome end at fusion junctions was also detected [33]. Similarly, when we tested the feasibility of a sequential FISH assay for the assessment of chromosome-end fusions [26] by analyzing G5 tert plants, we found that $47 \%$ of all identified fusions contained the signal from the same chromosome end. In the present study we describe the prevalent occurrence of the fusion of $2 \mathrm{R}$ chromosome ends in the double mutant, line K12 (Figure 6, Table 3). This can be explained by critically shortened 2R-telomere in early generation and by clonal propagation of this impairment through somatic tissues and germlines for succeeding generations. Albeit that the cause of the chromosome fusions in these various genetic backgrounds was rather different, a substantial proportion of the fusions of uncapped telomeres were accounted for by sister chromatid interactions.

\section{A possible role of NBS1 in the protection of telomeres}

We observed an increasing occurrence and higher frequency of anaphase bridges in double homozygotes than in wild-type or single tert mutants. From measuring the overall telomere length using terminal restriction fragment (TRF) analysis, we uncovered faster shortening of telomeres from individual chromosomes of the double mutants with respect to tert mutants (Figure 7). At the same time, the length of telomeres in nbs1 was unaffected and comparable with that of wild-type plants.

Substantial progress in the knowledge of RAD50 and MRE11 and their role in the DNA repair and telomere biology of Arabidopsis has been made while NBS1related data remain to be collected. From our experiments, we cannot gauge whether the synergism observed is due to a functional shortcoming or existence of a direct physical interaction of NBS1 with telomeres in plants. Possibly the first direct observation of the colocalization of the NBS1 protein with telomeres (TRF2 proteins) was found in HeLa cells during the $\mathrm{S}$ phase of the cell cycle, suggesting a possible role in telomere replication [14]. In the same year, interaction of NBS1 with TRF1 proteins was also observed in immortalized telomerase negative cells [13]. In experiments with cultured cells isolated from patients with the NBS1 syndrome, protein NBS1 was implicated as a positive regulator of the telomerase but the question remains as to whether the protein recruits this enzyme to the telomeres [34]. Using the cre-lox recombination system for the inactivation of TRF2 and NBS1 in mouse embryonal fibroblasts, the recruitment of NBS1 to dysfunctional telomeres was found to be cell cycle dependent [15]: the NHEJ was induced during G1 through ATM-dependent signalling, and, the NHEJ was repressed in G2 via a $5^{\prime}$ end-resection mechanism which produced an NHEJincompatible 3' overhang on replicated chromosomes.

In the TRF analysis, there were observed two different patterns, smeared signals for both, wild-type and nbs 1 plants representing various telomere lengths, and a number of individual bands representing single telomeres for plants with shortened telomeres (Figure 7). These discrete bands are hallmarks of telomere shortening [31] and provide evidence that shortened telomeres are clonally propagated in plant tissues and/or consecutive generations. Gross developmental defects of Arabidopsis plants and abundant end-to-end chromosome fusions in atm/tert mutants prove that all cells in the plant embryo inherit a critically shortened telomere [33]. Although analyses showed that the short telomere arose as a consequence of a large telomere rapid deletion (TRD) event, FISH analysis revealed that fused chromosomes exhibited overrepresented single chromosome end. Notably, in our tert/nbs 1 mutants, line $\mathrm{K} 12$, we detected overrepresented $2 \mathrm{R}$ chromosome ends at the fusion points in all generations analyzed by FISH (Figure 6). Interestingly, telomere shortening was detected from generation G2 (Figure 7A), far earlier than end-to-end chromosome fusions were detected in G3. This fact strongly supports the existence of a synergism between NBS1 and telomerase metabolism in Arabidopsis and excludes the possibility of the consequent effect of $n b s 1$ mutation on de-protected telomeres.

We did not address the issue of whether the deficiency of NBS1 alone was responsible for the observed phenotypes in double mutants or whether there was a disturbance in the proper assembly of the MRN complex with subsequent impact on the phenotypes. Hypomorphic mutation in the NBS1 gene in question, results in transcript truncated for the N-terminal portion thus lacking FHA and BRCT domains, whereas the C-terminal domain containing MRE11-binding and putative ATM-interaction motif sites are retained [23]. For this reason, the assembly of the MRN complex seems to be unaffected. The existence of phosphopeptide binding domains within the FHA and BRCT motifs in functional NBS1 renders multiple interactions with a plethora of proteins requiring phosphorylation to function in DNA-damage repair or telomere maintenance [35-37]. Thus, the dysfunction of the NBS1 can be entailed to the missing FHA/BRCT domain.

\section{Conclusions}

Direct physical contact/presence of NBS1 on telomeres and their function in telomere maintenance has been 
substantiated in the case of humans but not in plants. Understanding here is hampered by the fact that no shelterin counterparts had been described in plants to date. With our research we now provide indirect evidence of mutual interactions between NBS1 and telomeres. It is still unclear at this time whether protein truncation at the $\mathrm{N}$-terminal domain is responsible for the phenotypes observed in tert/nbs 1 mutants or if the disruption of certain unknown telomere-protecting pathways is responsible.

\section{Methods}

\section{Arabidopsis thaliana mutants}

Mutant plants (both Columbia background) were cultivated in an environmental growth chamber at $21^{\circ} \mathrm{C}$ under 16/8 h light/dark period. When cultured on agar plates, Murashige and Skoog medium including B5 vitamins (Duchefa) supplemented with $20 \mathrm{~g} / \mathrm{L}$ of sucrose in 0.8\% Plant Agar (Duchefa) was used and seeds germinated in permanent light. tert mutants were obtained from the laboratory of Dr. Karel Riha (described in [31]). nbs1 mutant line 570B09 was obtained from GABI-KAT [38]. Homozygous nbs 1 and tert plants were selected by PCR genotyping using T-DNA-specific and gene-specific primers for tert mutation LB6: GAACATCGG TCT CAATGCAA [31] and TERT6: CTAGGACATATCCATC AAGGGCT [31] and for nbs1 mutation NBSF: GGTT GTCCTTAATTCCGCTTG and 8409: ATATTGACCAT CATACTCATTGC. Homozygotes were crossed and from segregating F2 seedlings double homozygotes tert/nbs1, as well as wild-type, tert (two independent lines, 69-1 and 69-2), and nbs1 single mutants were selected and affirmed by PCR using TERT7: GAAAGGAAGCTGTATTGCAC GAA [31], TERT6 and NBSF and NBFR: GGCTGTATCC AGGAATTTCG, respectively. From double homozygotes, two independent lines were selected for further analyses, K12 and L2. These are further referred to as generation 1 (G1). With self pollination we obtained five consecutive generations, G2 to G6 until plants produced infertile seeds.

\section{DNA extraction and Terminal Restriction Fragment (TRF) analysis}

DNA was isolated according to [39] from inflorescences or young leaves 3 weeks after germination. TRF analysis was performed according to [40]. Briefly, DNAs were digested with Tru1I (Promega) overnight at $65^{\circ} \mathrm{C}$ and electrophoresed in $1 \%$ agarose. After Southern blotting, the membranes were hybridized with 32P-gamma-ATP end labelled (T3AG3)4 oligonucleotide. Radioactive signals were visualized on Typhoon FLA 9500 scanner and analyzed using ImageQuant software (GE Healthcare).

\section{Preparation of mitotic and meiotic chromosomes}

For the preparation of mitotic samples, whole terminal inflorescences were fixed in $3: 1$ mixture of ethanol and acetic acid. The inflorescences were washed with tap water and transferred to $10 \mathrm{mM}$ citrate buffer ( $\mathrm{pH} 4.5$ ). Pistils were excised from floral buds under dissection microscope and macerated using a mixture of 0.5\% Onozuka cellulase (SERVA Electrophoresis Ltd., Heidelberg, Germany) and 0.5\% Pectolyase (Sigma Chemical Co., Saint Louis, $\mathrm{MO}, \mathrm{USA}$ ) at $37^{\circ} \mathrm{C}$ in a moist chamber for $3 \mathrm{~h}$. Pistils were transferred into a drop of $60 \%$ acetic acid on slides and squashed under a cover-slip. After freezing of slides in liquid nitrogen and cover-slips removal, the slides were postfixed in 3:1 mixture of ethanol and acetic acid and air dried. Meiotic preparations were obtained after the same maceration procedure from anthers of immature flower buds (1-2 mm long).

\section{Anthers staining}

Anthers were fixed/stained in 1\% solution of carmine in $45 \%$ acetic acid for $30 \mathrm{~min}$ and then gently squashed under a cover-slip. Mature and healthy pollen grains stain in light purple.

\section{Bicolour FISH and labelling of probes}

As FISH probes for individual termini of Arabidopsis chromosomes, bacterial artificial chromosomes (BAC) clones were selected and using repeated FISH procedures the fusion points were assessed exactly as in [32]. BAC clones for the identification of each particular Arabidopsis chromosome end were F6F3, F5I6, F11L15, T4P13, F16M2, F6N15, T5J17, F7J8, and K9I9. These BACs were obtained from the Arabidopsis Biological Resource Center, Columbus, Ohio, USA. BAC DNAs were isolated from bacterial cultures using QIAGEN Plasmid Midi Kit (QIAGEN Inc., Valencia, CA, USA) exactly according to manufacturer recommendations. The probe for identifying the left arms of chromosomes 2 and 4 was the internal $2478 \mathrm{bp}$ (EcoR I) fragment of the 25SrRNA gene [41]. Probes were labelled either with SpectrumGreen-dUTP (Abbott Molecular, Illinois, USA) or Cy3-dUTP (GE Healthcare UK Ltd., Little Chalfont, England) using Nick Translation Mix (Roche Applied Science, Mannheim, Germany). Telomeric tracts were visualised by custom synthesized Cy3-tagged PNA probe CCCTAAACCCTAAA (Applied Biosystems, Bedford, MA, USA).

\section{FISH procedures and acquisition of images}

Microscope preparations were digested with $100 \mu \mathrm{g} / \mathrm{ml}$ in 2xSSC RNase A (Promega, Madison, WI, USA), for $1 \mathrm{~h}$ at $37^{\circ} \mathrm{C}$ and pepsin (Sigma, $50 \mu \mathrm{g} / \mathrm{ml}$ in $0.01 \mathrm{~N} \mathrm{HCl}$, $10 \mathrm{~min}, \mathrm{RT}$ ). Slides were post-fixed in $3.7 \%$ neutral formaldehyde. The hybridization mix contained $50 \mathrm{ng}$ of 
each labelled BAC DNA and/or of $20 \mathrm{ng}$ of labelled rDNA probe, $10 \%$ dextran sulphate, and $50 \%$ formamide in 2xSSC. Heat-denatured hybridization mixture was applied to slides, the slides were covered with cover-slips, and subjected to heat denaturation. After hybridization at $37^{\circ} \mathrm{C}$ overnight, the slides were stringently washed in $0.1 \mathrm{x} \mathrm{SSC}$ at $42^{\circ} \mathrm{C}$. Preparations were mounted in Vectashield (Vector Laboratories, Peterborough, UK) with $1 \mathrm{\mu g} / \mathrm{ml}$ 4',6-diamidino-2-phenylindole (DAPI, Sigma). For telomere PNA probe, the stringency of the hybridization was increased using $60 \%$ formamide in the probe mix. Microscope images were visualized using the Olympus AX 70 fluorescent microscope (Olympus, Tokyo, Japan) equipped with AxioCam MRm (Carl Zeiss Inc., Goettingen, Germany) camera and processed with ISIS imaging software (MetaSystems Ltd., Altlussheim, Germany).

\begin{abstract}
Abbreviations
ATM: Ataxia telangiectasia mutated; ATR: Ataxia telangiectasia and Rad 3 related; BAC: Bacterial artificial chromosome; BRCA1: Breast cancer 1 gene; BRCT: BRCA1 C terminus domain; DSB: Double strand break; FHA: Forkheadassociated domain; KU70: KU70 protein; MRE11: Meiotic recombination 11 gene; MRN: MRE11-RAD50-NBS1 complex; NBS1: Nijmegen breakage syndrome 1 gene; NHEJ: Non-homologous-end-joining; PNA: Peptide nucleic acid; RAD50: DNA repair protein; TERT: Telomerase reverse transcriptase; TRD: Telomere rapid deletion; TRF: Terminal restriction fragment analysis; TRF1: Telomeric repeat binding factor 1; TRF2: Telomeric repeat binding factor 2; XRS2: X-rays sensitive, yeast DNA repair protein.
\end{abstract}

\section{Competing interests}

The authors declare that they have no competing interests.

\section{Authors' contributions}

JS conceived the concept, LN and JS performed the experiments, and JS and $L N$ wrote the paper. Both authors read and approved the final manuscript.

\section{Acknowledgements}

We are indebted to Dr. Karel Riha, Gregor Mendel Institute of Molecular Plant Biology, Vienna, Austria for providing us seeds of A. thaliana tert mutants. This work was supported from the Czech Science Foundation, Grant No. P501/12/G090

Received: 30 April 2012 Accepted: 10 September 2012

Published: 17 September 2012

\section{References}

1. de Lange T: Shelterin: the protein complex that shapes and safeguards human telomeres. Gene Dev 2005, 19:2100-2110.

2. Blackburn EH: Telomeres and telomerase: their mechanisms of action and the effects of altering their functions. FEBS Lett 2005, 5579:859-862.

3. Verdun RE, Karlseder J: Replication and protection of telomeres. Nature 2007, 447:924-931.

4. Palm W, de Lange T: How Shelterin Protects Mammalian Telomeres. Annu Rev Genet 2008, 42:301-334.

5. Watson JM, Riha K: Comparative biology of telomeres: Where plants stand. FEBS Lett 2010, 584:3752-3759.

6. Lamarche BJ, Orazio NI, Weitzman MD: The MRN complex in double-strand break repair and telomere maintenance. FEBS Lett 2010, 584:3682-3695.

7. Stacker TH, Petrini JHJ: The MRE11 complex: starting from the ends. Nat Rev Mol Cell Bio 2011, 12:90-103.

8. Hopfner KP, Craig L, Moncalian G, Zinkel RA, Usui T, Owen BA, Karcher A Henderson B, Bodmer JL, McMurray CT, Carney JP, Petrini JH, Tainer JA: The Rad50 zinc-hook is a structure joining Mre11 complexes in DNA recombination and repair. Nature 2002, 418:562-566.
9. Amiard S, Charbonnel C, Allain E, Depeiges A, White Cl, Gallego ME: Distinct Roles of the ATR Kinase and the Mre11-Rad50-Nbs1 Complex in the Maintenance of Chromosomal Stability in Arabidopsis. Plant Cell 2010, 22:3020-3033.

10. Ueno M, Nakazaki T, Akamatsu Y, Watanabe K, Tomita K, Lindsay HD, Shinagawa H, Iwasaki H: Molecular Characterization of the Schizosaccharomyces pombe nbs $1^{+}$Gene Involved in DNA Repair and Telomere Maintenance. Mol Cell Biol 2003, 23:6553-6563.

11. Zhang Y, Zhou J, Lim CUK: The role of NBS1 in DNA double strand break repair, telomere stability, and cell cycle checkpoint control. Cell Res 2006, 16:45-54.

12. Zhu J, Petersen S, Tessarollo L, Nussenzweig A: Targeted disruption of the Nijmegen breakage syndrome gene NBS1 leads to early embryonic lethality in mice. Curr Biol 2001, 11:105-109.

13. Wu G, Lee W-H, Chen P-L: NBS1 and TRF1 Colocalize at Promyelocytic Leukemia Bodies during Late S/G2 Phases in Immortalized Telomerasenegative Cells. J Biol Chem 2000, 275:30618-30622.

14. Zhu XD, Kuster B, Mann M, Petrini JH, de Lange T: Cell-cycle-regulated association of RAD50/MRE11/NBS1 with TRF2 and human telomeres. Nat Genet 2000, 25:347-352.

15. Dimitrova N, de Lange T: Cell Cycle-Dependent Role of MRN at Dysfunctional Telomeres: ATM Signaling-Dependent Induction of Nonhomologous End Joining (NHEJ) in G1 and Resection-Mediated Inhibition of NHEJ in G2. Mol Cell Biol 2009, 29:5552-5563.

16. Zellinger B, Riha K: Composition of plant telomeres. Biochim Biophys Acta 2007, 1769:399-409.

17. Siroky J: Cytogenetics for the study of telomere function in plants. Cytogenet Genome Res 2008, 122:374-379.

18. Bleuyard J-Y, Gallego ME, White Cl: Meiotic defects in the Arabidopsis rad50 mutant point to conservation of the MRX komplex function in early stages of meiotic recombination. Chromosoma 2004, 113:197-203.

19. Vannier JB, Depeiges A, White C, Gallego ME: Two roles for Rad50 in telomere maintenance. EMBO J 2006, 25:4577-4585.

20. Puizina J, Siroky J, Mokros P, Schweizer D, Riha K: Mre11 deficiency in Arabidopsis is associated with chromosomal instability in somatic cells and Spo11-dependent genome fragmentation during meiosis. Plant Cell 2004, 16:1968-1978.

21. Heacock M, Spangler E, Riha K, Puizina J, Shippen DE: Molecular analysis of telomere fusions in Arabidopsis. multiple pathways for chromosome end-joining. EMBO J 2004, 23:2304-2313.

22. Akutsu N, lijima K, Hinata T, Tauchi H: Characterization of the plant homolog of Nijmegen breakage syndrome 1: Involvement in DNA repair and recombination. Biochem Biophys Res Co 2007, 353:394-398.

23. Waterworth WM, Altun C, Armstrong SJ, Roberts N, Dean PJ, Young K, Weil CF, Bray CM, West CE: NBS1 is involved in DNA repair and plays a synergistic role with ATM in mediating meiotic homologous recombination in plants. Plant J 2007, 52:41-52.

24. Riha K, McKnight TD, Griffing LR, Shippen DE: Living with genome instability: plant responses to telomere dysfunction. Science 2001, 291:1797-1800.

25. Newcombe RG: Two-Sided Confidence Intervals for the Single Proportion: Comparison of Seven Methods. Stat Med 1998, 17:857-872.

26. Mokros P, Vrbsky J, Siroky J: Identification of chromosomal fusion sites in Arabidopsis mutants using sequential bicolour BAC-FISH. Genome 2006, 49:1036-1042

27. Gallego ME, White Cl: DNA repair and recombination functions in Arabidopsis telomere maintenance. Chromosome Res 2005, 13:481-491.

28. Bundock P, Hooykaas P: Severe developmental defects, hypersensitivity to DNA-damaging agents, and lengthened telomeres in Arabidopsis MRE11 mutants. Plant Cell 2002, 14:2451-2462.

29. Gallego ME, White Cl: RAD50 function is essential for telomere maintenance in Arabidopsis. P Natl Acad Sci USA 2001, 98:1711-1716.

30. Gallego ME, Jeanneau M, Granier F, Bouchej D, Bechtold N, White Cl: Disruption of the Arabidopsis RAD50 gene leads to plant sterility and MMS sensitivity. Plant J 2001, 25:31-41.

31. Fitzgerald MS, Riha K, Gao F, Ren SX, McKnight TD, Shippen DE: Disruption of the telomerase catalytic subunit gene from Arabidopsis inactivates telomerase and leads to a slow loss of telomeric DNA. P Natl Acad Sci USA 1999, 96:14813-14818

32. Siroky J, Zluvova J, Riha K, Shippen DE, Vyskot B: Rearrangements of ribosomal DNA clusters in late generation telomerase-deficient Arabidopsis. Chromosoma 2003, 112:116-123. 
33. Vespa L, Ross T, Warrington RT, Mokros P, Siroky J, Shippen DE: ATM regulates the length of individual telomere tracts in Arabidopsis. $P$ Natl Acad Sci USA 2007, 104:18145-18150.

34. Ranganathan V, Heine WF, Ciccone DN, Rudolph KL, Wu X, Chang S, Hai H, Ahearn IM, Livingston DM, Resnick I, Rosen F, Seemanova E, Jarolim P, DePinho RA, Weaver DT: Rescue of a telomere length defect of Nijmegen breakage syndrome cells requires NBS and telomerase catalytic subunit. Curr Biol 2001, 11:962-966.

35. Lim DS, Kim ST, Xu B, Maser RS, Lin J, Petrini JH, Kastan MB: ATM phosphorylates p95/nbs1 in an S-phase checkpoint pathway. Nature 2000, 404:613-617.

36. Lee JH, Paull TT: ATM activation by DNA double-strand breaks through the Mre11-Rad50-Nbs1 complex. Science 2005, 308:551-554.

37. Williams GJ, Lees-Miller SP, Tainer JA: Mre11-Rad50-Nbs1 conformations and the control of sensing, signaling, and effector responses at DNA double-strand breaks. DNA Repair 2010, 9:1299-1306.

38. Rosso MG, Li Y, Strizhov N, Reiss B, Dekker K, Weisshaar B: An Arabidopsis thaliana T-DNA mutagenized population (GABI-Kat) for flanking sequence tag-based reverse genetics. Plant Mol Biol 2003, 53:247-259.

39. Dellaporta SL, Wood J, Hicks JB: A plant DNA minipreparation. Version II. Plant Mol Biol Rep 1983, 1:19-21.

40. Watson JM, Shippen DE: Telomere rapid deletion regulates telomere length in Arabidopsis thaliana. Mol Cell Biol 2007, 27:1706-1715.

41. Kiss T, Kiss M, Solymosy F: Nucleotide sequence of a 25S rRNA gene from tomato. Nucleic Acids Res 1989, 17:796.

doi:10.1186/1471-2229-12-167

Cite this article as: Najdekrova and Siroky: NBS1 plays a synergistic role with telomerase in the maintenance of telomeres in Arabidopsis thaliana. BMC Plant Biology 2012 12:167

\section{Submit your next manuscript to BioMed Central and take full advantage of:}

- Convenient online submission

- Thorough peer review

- No space constraints or color figure charges

- Immediate publication on acceptance

- Inclusion in PubMed, CAS, Scopus and Google Scholar

- Research which is freely available for redistribution 